\title{
Changes in gait detected by three-dimensional motion analysis in patients with acoustic neuroma
}

\begin{abstract}
Many patients with acoustic neuroma experience hearing loss, tinnitus, and equilibrium disturbance. Gait studies performed using tactile sensors placed under the feet have indicated that an acoustic neuroma causes unstable gait, even though this cannot be detected by visual observation. Three-dimensional motion analysis is a useful tool to assess gait. The purpose of this study was to use a three-dimensional motion analysis system to quantify the spatiotemporal and kinematic parameters of gait in patients with acoustic neuroma. Seventeen patients with unilateral acoustic neuroma and seven age-, height-, and foot-size-matched healthy subjects participated. Subjects were asked to walk 4 meters in eyes-open and eyes-closed conditions. Gait speed, stride length, stride duration, head movements in the vertical direction and yaw, pitch, and roll dimensions, gait deviation, forefoot motion, and ankle plantar flexion angle at heel strike were quantified. Patients with acoustic neuroma had slower gait with wider step width and shorter stride length along with greater head movement in pitch and roll than controls. In addition, patients with acoustic neuroma had lower ankle plantar flexion angle at heel strike than controls, especially in the eyes-closed condition. These characteristics of gait increase our understanding of the pathophysiological changes caused by acoustic neuroma.
\end{abstract}

Keywords: gait analysis, acoustic neuroma, three-dimensional motion analysis, ankle plantar flexion
Volume 2 Issue 4 - 2015

\author{
Aboshanif Mohamed, Kazuo Ishikawa, \\ Yoshiaki Itasaka, Eigo Omi, Koh Koizumi, \\ Shinsuke Suzuki, Kohei Honda \\ Department of Otorhinolaryngology, Akita University, Japan
}

Correspondence: Kazuo Ishikawa, Department of Otorhinolaryngology, Head \&Neck Surgery, Akita Graduate School of Medicine, I- I-I, Hondo, Akita 0 I0-8543, Japan, Tel +8 I I88846171, Fax +8| 188362622

Email ishioto@med.akita-u.ac.jp

Received: March25, 20I5 | Published: April 30, 2015

\section{Introduction}

Human gait is a whole-body movement that is controlled at many levels. ${ }^{1}$ The peripheral vestibular system plays an important role in normal gait performance. ${ }^{2}$ Unilateral vestibular lesions can cause the body to move toward the lesioned side during locomotion and this becomes evident when visual input is removed. This shift in locomotion can clearly be seen in patients with large acoustic tumor or patients in the acute phase of a peripheral vestibular dysfunction such as vestibular neuronitis or Meniere's disease.

In addition to this gait shift, the stability of gait performance is altered in patients with unilateral vestibular lesion. The coefficient of variation of gait-phase-related variables such as stance and swing was greater than in healthy controls, ${ }^{3}$ reflecting the vestibular end-organ's contribution toward the control of phase of gait. ${ }^{4}$ And the magnitude of the coefficient of variation may reflect the size of the lesion in the peripheral and central vestibular system.,

However, as gait is a whole-body motor task, some lesions in the gait control system should result in abnormal performance at various parts of the body movement such as head, shoulders, legs, knees, feet and so on. Therefore, we performed three-dimensional whole-body gait analysis in patients with acoustic neuroma AN that originated from the vestibular nerve.

\section{Materials and methods}

Subjects for the present study were 17 patients seven males and 10 females with AN that was diagnosed by neurootological examinations and MRI with contrast enhancement. The average age of patients was $64.1 \pm 11.1$ years and the average height was $163.7 \pm 7.8 \mathrm{~cm}$. The tumor was on the right side in 11 patients and the left side in 6 patients. The tumor was less than $2 \mathrm{~cm}$ from porus acusticus internus in 13 patients and more than $2 \mathrm{~cm}$ from porus acusticus internus in four patients. Seven age-, foot-size-, and height-matched healthy subjects three males and four females also participated as controls. The average age of controls was $63.4 \pm 4.9$ years and the average height was $161.2 \pm 8.7 \mathrm{~cm}$.

Three-dimensional whole-body movement analysis was performed using the analysis system shown in Figure 1. Fifteen reflective markers were placed on the body at the following locations: vertex, forehead, inion, and bilateral shoulder, elbow, wrist, knee, heel, and toe. Subjects were asked to walk for a distance of about 4 meters with their eyes open or closed, and then turn around and walk back to the starting position. This enabled us to measure both sides of the body with the two-camera system. Three-dimensional analysis was performed over a 2 cubic meters space located at the center of the 4-m walkway (Figure 1). Spatiotemporal and kinematic variables, including walking speed, stride length, stride duration, stance phase ratio, step width, forefoot motion, ankle planter flexion angle, head movements in roll, pitch and yaw, and walking deviation, were calculated. All variables were compared across groups AN, control using a Student's t-test. P values less that 0.05 were considered statistically significant.

\section{Results}

Gait speed was significantly slower in the AN group than in the control group in the eyes-open condition. There was a similar trend in the eyes-closed condition, but this was not significant (Figure 2A). Stride length was significantly shorter in the AN group than in the control group in eyes-open and eyes-closed conditions (Figure 2B). There was no significant group difference in stride time (Figure 2C).

Neither the lowest position of the toe during swing nor the highest position of the toe during swing showed a group difference (Figure 3 (A \& B)). Step width was wider in the eyes-closed condition than in the eyes-open condition in both groups, but was not significantly different between the two groups (Figure 3C). Ankle plantar flexion angle at heel strike was smaller in the AN group than in the control 
group under both the eyes-open and the eyes-closed condition (Figure 4A). Moreover, ankle plantar flexion angle at heel strike tended to be smaller in the larger tumor group than in the smaller tumor group, especially with eyes closed (Figure 4B).

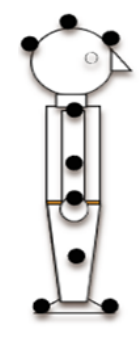

a)

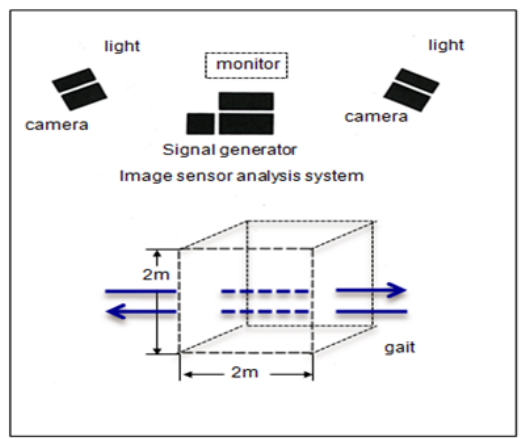

b)
Figure I Block diagram of the three-dimensional gait analysis system.

a) Markers were placed on the vertex, forehead, and inion and bilaterally on the, shoulder, elbow, wrist, knee, heel, and toe.

b) Gait was analyzed over a 2-m3 space at the center of a 4-m walkway. Black circles: positions of reflective markers.Arrows: direction of gait.

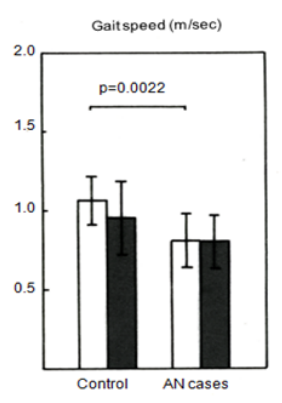

a)
Stride length $(m)$

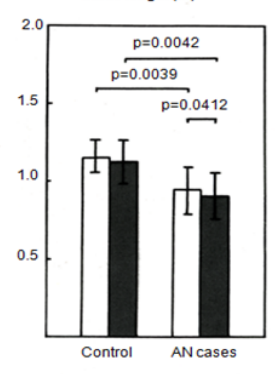

Gaitwith eyes open

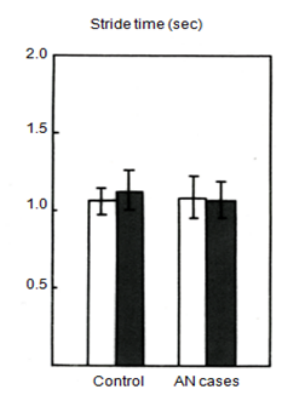

Figure 2 a) Gait speed, b) Stride length, and c) Stride time. Gait speed was lower in the AN group than in the control group in the eyes-open condition; however no difference was present in the eyes-closed condition. Stride length was shorter in the AN group than in the control group. Stride time was similar in the two groups. White: gait with eyes open. Black: gait with eyes closed.

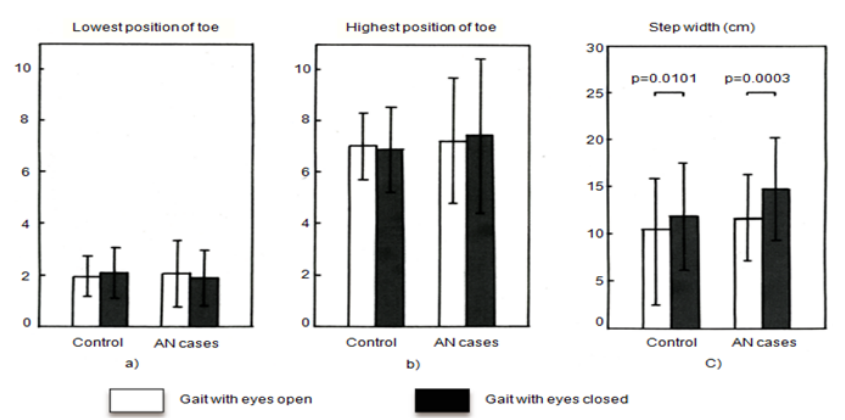

Figure 3 a, b) Toe swing and c) Step width. The lowest and highest positions of the toe during swing were similar in the two group. Step width was wider in the eyes-closed condition than in the eyes-open condition in both groups, but was similar in the two groups.

Head movement in the yaw plane was similar in the two groups. However, head movement in the pitch and roll planes was significantly greater in the AN group, especially in the eyes-closed condition
(Figure 5). Vertical movement of the vertex during locomotion was similar in the two groups, but horizontal deviation was greater in the AN group (Figure 6).
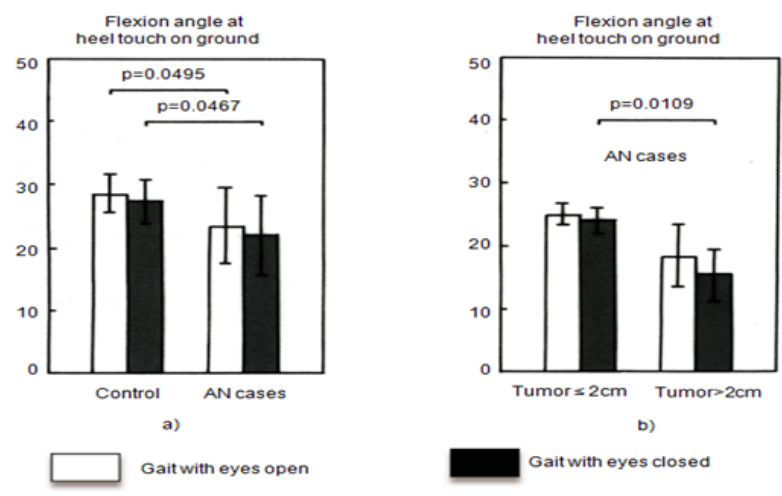

Figure 4 Ankle planter flexion angle at heel strike.

a) Flexion angle was smaller.

b) In the AN group than in the control group, and was even smaller in patients with large tumors.

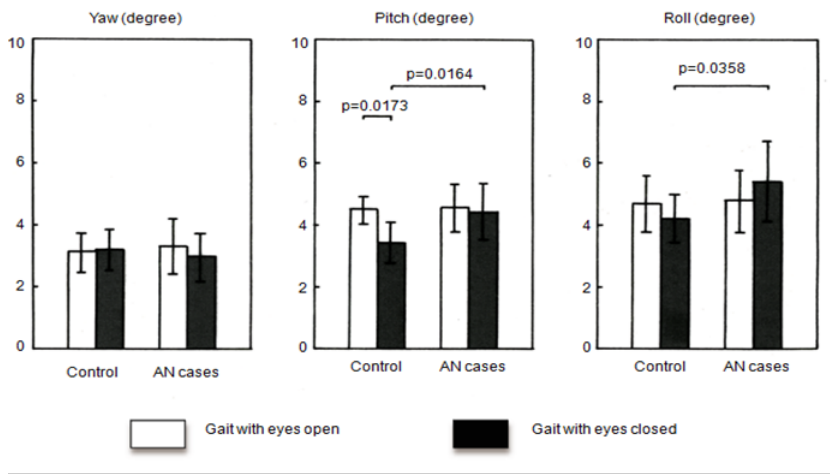

Figure 5 Head movement during locomotion. Pitch and roll movements were greater in patients with AN than in controls, especially in the eyes-closed condition.
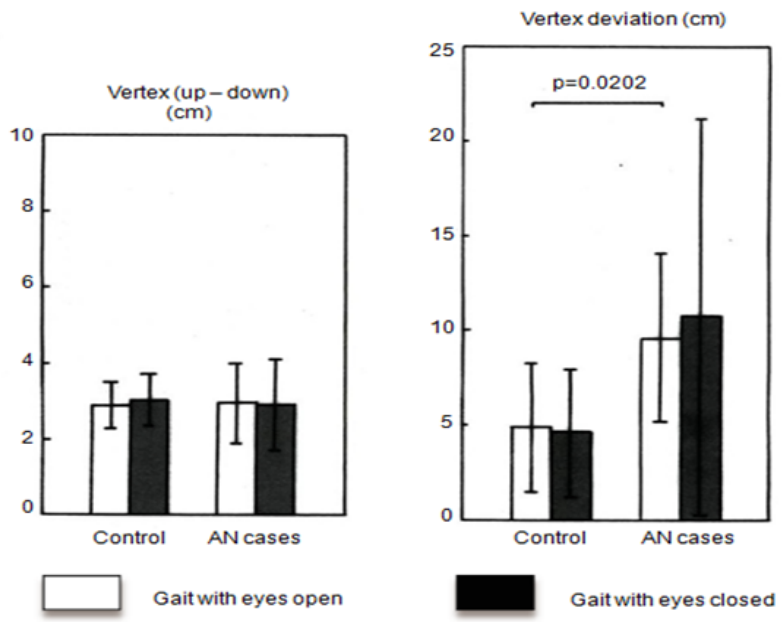

Figure 6 Head movement in the vertical direction, and gait deviation. Head movement in the vertical direction was similar in AN patients and controls; however, gait deviation depicted by vertex deviation was greater in AN patients than in controls. 


\section{Discussion}

Functional disorder of the peripheral vestibular system caused by disease results in abnormal eye movements, space disorientation, ${ }^{7}$ and postural and locomotor abnormalities. ${ }^{8}$ Also, visual input is important for controlling locomotor performance as feed forward information in conjunction with stabilizing a space related with visual suppression, especially in pathological cases. ${ }^{9,10}$

It is well known that locomotor abnormalities caused by a peripheral lesion are characterized by gait deviation and slow gait. ${ }^{11}$ However, detailed gait analysis has not yet been fully performed. As for the postural change, head deviation along with neck torsion can be caused by unilateral labyrinthectomy. ${ }^{12}$ The degree of these abnormalities can be influenced by the severity of the lesion. When the lesion is mild, the abnormalities can also be mild, and sometimes cannot be identified by visual observation. However a detailed motion analysis using the recent up-dated methods, could detect minor changes.

The use of tactile sensors during gait analysis in patients with vestibular system disorders provides important information about vestibular pathophysiology. ${ }^{13}$ In a previous study we used tactile sensors to detect a gait abnormality in patients with small unilateral AN and seemingly normal gait. This abnormality was an increment in the coefficient of variation of gait-phase-related variables. ${ }^{3}$ This was in line with a previous report that the peripheral vestibular system plays an important role in the control of gait phase. ${ }^{2}$ However, so far, no detailed whole-body analysis of gait has been performed in patients with AN.

In the present study we placed markers on the head, trunk, arms, waist and feet of the subjects and measured whole-body movements during gait performed with eyes open and eyes closed. Patients with AN had a slower gait with wider step width and shorter stride length, especially when gait was performed without visual input. These minor but significant changes likely reflect the presence of a defense mechanism equipped in our central nervous system against minor functional change which is not clear to the observer. In addition, ankle plantar flexion angle at heel strike was significantly lower in AN patients, especially when gait was performed with eyes closed. This change was even more pronounced in patients with large tumors. These changes were observed in the absence of differences in gait speed, and the decreased ankle planter flexion angle at heel strike could be caused by the presence of the tumor, probably due to a decreased functional level of the lateral vestibulo-spinal tract. ${ }^{14}$

Head movement in pitch and roll was slightly but significantly greater in AN patients than in controls, especially in the eyes-closed condition, indicating that visual cues are important for head stability during walking and that asymmetric otolith function caused by the tumor could play some role in head stability. Recently, it has become possible to evaluate the function of the two otolith organs separately. ${ }^{15}$ The contribution of otolith function toward head stability during gait should further be examined in more detail.

Despite our expectation, the height of the toe during swing was similar in patients and controls. This height could be kept equal even when walking on a slope. ${ }^{7}$ A change in the height of the toe, especially at lowest toe swing, may lead to stumbles during gait, and the height of the toe is believed to be one of the essential factors for smoothness gait. Therefore in patients with a very large tumor, a change in the height of the toe during swing may cause falls, and this should be clarified in future studies.

The greater gait deviation observed in patients with a tumor could indicate space disorientation induced by peripheral vestibular dysfunction. Usually this kind of gait deviation drifts the body toward the lesioned side, although there was greater variability in gait deviation during gait with eyes closed than during gait with eyes open, which mostly could be affected by the state of vestibular compensation in each case.

\section{Conclusion}

A detailed whole-body gait analysis could delineate gait abnormalities in patients with AN. However, we have to know that most of those findings can be concealed by visual input especially when abnormalities are mild due to recalibration of the postural control system by the use of visual information. Further studies are required to obtain details as to the relationship between head stability and utricular and/or saccular function in addition to the other diseasespecific gait abnormalities that could lead to early detection of disease and prevention of falls.

\section{Acknowledgments}

None.

\section{Conflicts of interest}

The authors declare that there are no conflicts of interest.

\section{Funding details}

None.

\section{References}

1. Mori S. Integration of posture and locomotion in acute decerebrate cats and in awake, freely moving cats. Prog Neurobiol. 1987;28(2):161-195.

2. Kanaya T. Functional roles played by lateral vestibular neurons during controlled locomotion in the mesencephalic cat. Nihon Jibiinkoka Gakkai Kaiho. 1984;87(5):667-680.

3. Ishikawa K, Edo M, Yokomizo M, et al. Comparative study on gait abnormality in patients with vestibular neuronitis and patients with large acoustic neuroma. Acta Otolaryngol Suppl. 1995;519:197-200.

4. Mori S, Matsuyama K, Takakusaki K, et al. The behaviour of lateral vestibular neurons during walk, trot and gallop in acute precollicular decerebrate cats. Prog Brain Res. 1988;76:211-220.

5. Ishikawa K, Edo M, Yokomizo M, et al. Characteristics of human gait related variables in association with vestibular system disorders. Acta Otolaryngol. 1995;(Supp1520Pt1):199-201.

6. Ishikawa K, Wang Y, Wong WH, et al. Gait instability in patients with acoustic neuroma. Acta Otolaryngol. 2004;124(4):486-489.

7. Cohen HS. Vestibular disorders and impaired path integration along a linear trajectory. J Vestibular Res. 2000;10(1):7-15.

8. Ishikawa Kazuo, Cao ZW, Wang Y, et al. Dynamic locomotor function in normals and patients with vertigo. Acta Otolaryngol. 2001;121(2):241-244.

9. Deshpande N, Patla AE. Dynamic visual-vestibular integration during goal directed human locomotion. Exp Brain Res. 2005;166(2):237-247. 
10. Reynolds RF, Day BL. Visual guidance of the human foot during a step. J Physiol. 2005;569(Pt 2):677-684.

11. Igarashi M, Ishikawa K. Post-labyrinthectomy balance compensation with preplacement of cerebellar vermis lesion. Acta Otolaryngol. $1985 ; 99(3-4): 452-458$.

12. Schaefer KP, Meyer DL. Aspects of vestibular compensation in guinea pigs. Lesion-induced neural plasticity in sensorimotor system. 1981;197-207.
13. Angunsri N, Ishikawa K, Yin M, et al. Gait instability caused by vestibular disorders-analysis by tactile sensor. Auris Nasus Larynx. 2011;38(4): 462-468.

14. Jay MG, Victor JW, Kathleen EC, et al. The vestibulospinal system and postural control. In The vestibular system: a sixth sense, Oxford university press, Inc, 2012. p. 280-312.

15. Curthoys IS. The interpretation of clinical tests of peripheral vestibular function. Laryngoscope. 2012;122(6):1342-1352. 